I thank Miss Ellen Gundermann and Mr. C. E. Heiles for carrying out the reduction of the obserrational data.

National Radio Astronomy Observatory *,

$$
\text { F. D. Drake }
$$
Groen Bank, West Virginia.

* Operated by Associated Universities, Inc., under contract with the U.S. National Science Foundation.

${ }^{I}$ Cook, J. J., Gross, L. G., Bair, M. E., and Arnold, C. B., Nature, $188,393(1960)$.

? Drake, F. D., and Ewen. H. I., Proc. Inst. Rad. Eng. 46, 53 (1958).

${ }^{3}$ Drake, F. D., and Hvatum, H., Astro. J., 64, 329 (1959).

+ Radhakrishnan, V., and Roberts, J. A., Phys. Rev. Letters, 4, 493 (1960)

Drake, F. D. (following communication).

6 Pierce, B., Astro. J., 2, 161 (1853).

Pettit, E., Planets and Satellites, edit. by Kuiper, G. P., 400 (Univ. Chicago Press, 1961).

${ }^{8}$ Sagan, C., Rad. Res., 15, 174 (1961).

Sloanaker, R. M., and Boland, J. W., Astrophys. J., 133, 649 (1961).

${ }^{10}$ Drake, F. D. (in preparation).

\section{0-cm, Observations of Venus near Superior Conjunction}

Previous extended observations ${ }^{1}$ of Venus at 10-cm wave-length have given evidenco for only a small variation in the mean disk equivalent blackbody temperature $T_{B B}$ with Venus phase angle $i$. These observations were made during a period surrounding inferior conjunction, and did not extend to small phase angles. Thus the disk temperature for small $i$ could be inferred only from an uncomfortably large extrapolation of the uncertain function $T_{B B}(i)$ derived from data containing significant random, and perhaps systematic, errors. These circumstances have made it desirable to observe Venus, if possible, near superior conjunction. This is difficult because Venus is then at maximum distance, leading to minimum signal-to-noise ratios, and the planet also appears close to the Sun, a powerful radiator which can contaminate the observations by introducing significant radiation into the antenna side-lobes.

In an attempt to measure $T_{B B}$ near superior conjunction, Venus was observed for $330 \mathrm{~h}$ on 44 days during the period March 9-May 13, 1962. The equipment used was the $85-\mathrm{ft}$. telescope of the National Radio Astronomy Observatory and a 10.0em travelling wave tube radiometer ${ }^{2}$ with digital data recording devices; this was the same equipment used to obtain the previous extended set of data ${ }^{1}$. Observations consisted of 30-sec observations made with the antenna pointed directly at the planet, alternating with 30 -sec observations made with the antenna pointed $30^{\prime}$ to one side of the planet. These latter comparison positions were arranged symmetrically north, south, east, and west of the planet. For purposes of calibration, observations of an argon noise tube were made at frequent intervals. A directional coupler introduced the noise tube output power into the radiometer so as to avoid physical changes in the radiometer in the course of the observations. The over-all telescope system was calibrated several times each day by observing the radio source $04 N 3 A$ in the same manner as Venus. From these data, the ratio of the intensity of Venus to that of $04 N 3 A$ was calculated, which gives directly the Venus flux density, if the flux density from $04 N 3 A$ is known. The adoptod value of the $04 N 3 A$ flux density was $23 \cdot 7\left(10^{-26}\right) \mathrm{W} / \mathrm{m}^{2} / \mathrm{c} / \mathrm{s}^{1,3}$. The standard black-body radiation formulæ and the ephemeris planetary semi-diameter then led directly to a value for $T_{B B}$. An IBM 1620 computer, which road directly the punched-tape records prepared at the telescope, was used in the reductions. These observation, calibration, and reduction procedures are identical to those used previously. Thus the result obtained here is homogenoous with the previous data. In particular, since the primary calibration standard is the flux from $04 N 3 A$, there should be no systematic differences in the calibration of $T_{B B}$ between the present and previous sots of data.

Inspection of the 44 daily mean values of $T_{B B}$ showed that they were distributed in the expected random way about a mean value, except for: (1) two values which deviated widely from the general run of values; (2) all data obtained during April 6-23, which deviated non-randomly from the mean by large amounts. An analysis of these latter divergent data showed that values of $T_{B B}$ based on observations using comparison points east and west of the planet differed significantly from the values obtained using north and south comparison points. This can only be due to an interfering source of radiation. and the length of time over which this problem persisted shows that the source can be only the Sun. Consequently, all points from this period were discarded. The other two divergent points were discarded on the basis of Pierce's criterion ${ }^{4}$. This left 36 of the original 44 days' observations, representing about 250 observing hours. The mean value of $T_{B B}$ obtained from these accepted data was:

$$
T_{B B}=610 \pm 55(\mathrm{~m} \cdot \theta \cdot)^{\circ} \mathrm{K}
$$

The range of $i$ spanned by the observations was $14^{\circ}<i<37^{\circ}$, with the mean value of $i$ being $i=25^{\circ}$. The previous results gave $T_{B B}=629+39$ $\cos \left(i-17^{\circ}\right)^{\circ} \mathrm{K}$ for the range of $i$ of concern here. This relation predicts $T_{B B}=660^{\circ} \mathrm{K}$ at $i=25^{\circ}$. Thus the present and previous results are mutually consistent. They are strong evidence that the $10-\mathrm{cm}$ phase effect is very small, nearly that given previously, and that there is little surface temperature difference between the illuminated and dark hemispheres of Venus.

I thank Miss Ellen Gundormann and Mr. C. E. Heiles for carrying out the data reductions. F. D. Drake

National Radio Astronomy Observatory*, Green Bank, West Virginia.

* Operated by Associated Universities, Inc., undir contract with the U.S. National Science Foundation.

1 Drake, F. D., Pub. Nat. Radio Astro. Obs., 1, No. 11, 165 (1962), 2 Drake, F. D., and Ewen, H. I., Proc. Inst. Rad. Eng., 46, 53 (1958). $s$ Heeschen, D. S., and Meredith, B. L., Pub. Nat. Radio Astro. Obs., $1,8,121$ (196i). 4 Pierce, B., Astrophys. J., 2, 161 (1853).

\section{The Lyman- $\alpha$ Problem and the Geocoma Hypothesis}

IN a recent review article, Donahue' has discussed the various hypotheses put forward to explain the observed Lyman- $\alpha$ radiation ${ }^{2,3}$ in the night sky. The observod radiation is apparently solar Lyman- $\alpha$ radiation scattered by neutral hydrogen. There are three hypotheses concerning the location of the hydrogen: namely, the interplanetary hypothesis ${ }^{3}$; tho geocorona hypothesis ${ }^{4,5}$ (origin in a thin, essentially spherical shell with multiple scattering important); and the geocoma hypothesis ${ }^{6,7}$ (origin in a comet-like distribution at distances greater than some $5-10 R_{E}$, single scattering important). It is felt that the review ${ }^{1}$ is generally not unfavourable to the 